\title{
La ergometría en la vigilancia de la salud en una población laboral de extinción de incendios
}

\author{
The ergometry in the vigilance of the \\ health in firemen
}

\author{
Antonio Ares Camerino \\ Unidad de Salud Laboral. Consorcio de Bomberos de la Provincia de Cádiz \\ Diputación Provincial de Cádiz. España \\ Jesús Oneto Otero \\ Unidad de Salud Laboral. Consorcio de Bomberos de la Provincia de Cádiz \\ Unidad de Cardiología. Clínica de La Salud. Cádiz. España

\section{Eduardo M. Morentin} \\ Unidad de Salud Laboral. Consorcio de Bomberos de la Provincia de Cádiz \\ Unidad de Cardiología. Clínica de La Salud. Cádiz. España \\ Jesús Mora Vicente \\ Unidad de Salud Laboral. Consorcio de Bomberos de la Provincia de Cádiz \\ Escuela Universitaria. Universidad de Cádiz. España \\ Beatriz Sainz Vera \\ Unidad de Salud Laboral. Consorcio de Bomberos de la Provincia de Cádiz \\ Hospital Clínico de Puerto Real. Cádiz. España \\ Maria L. Soto Pino \\ Unidad de Salud Laboral. Consorcio de Bomberos de la Provincia de Cádiz \\ Diputación Provincial de Cádiz. España \\ Correspondencia: \\ Antonio Ares Camerino. Diputación Provincial de Cádiz. \\ Plaza de España s/n \\ 11007 Cádiz. España \\ Teléfono: 956240124 \\ Fax: 956228839 \\ email: aares@dipucadiz.es
}

Resumen

La indicación de la ergometría en determinados colectivos de personas trabajadoras está recogida con la categoría IIa (opinión a favor de su utilidad) de la American Hearth Association. Los protocolos de vigilancia en salud laboral (Ministerio de Sanidad y Consumo) no recogen la realización de ergometrías.

Objetivo: analizar los datos de ergometrías realizadas a 31 bomberos mayores de 44 años.

Método: Se aplico protocolo BRUCE sobre tapiz rodante. Se analizaron los siguientes datos: edad, consumo de tabaco, frecuencia cardiaca basal, PR, eje QRS, frecuencia cardiaca submáxima, TAS/TAD basal, TAS/TAD máxima, capacidad funcional, tiempo de duración de la prueba, colesterol total, HDL, LDL y glucemia. Se analizó el riesgo cardiovascular

Resultados: La media de edad fue de 48,82 años. El 29,03\% eran fumadores. Los datos analítico fueron: glucemia 85,37 mg/100 cc, colesterol total 205 mg/100 cc, HDL 53,03 mg/100 cc, LDL 151,07 mg/100 cc. Buena respuesta al ejercicio físico. La capacidad funcional fue de 12,87 Mets, y la duración de 11,46 minutos. 
Conclusión: Incluir la ergometría en los protocolos de vigilancia de la salud para determinados colectivos de trabajadores

(Med Segur Trab (Internet) 2009; 55 (216): 56-64)

Palabras claves: ergometria, diagnóstico, población, bomberos

Abstract

The indication of the ergometry in certain groups of hard-working persons is gathered by the category IIa (opinion in favour of its utility) of the American Hearth Association. The protocols of alertness in labour health (Ministry of Health and Consumption) do not gather the accomplishment of ergometry.

Objective: to analyze the information of ergometry realized to 3144 -year-old major firemen.

Method: Applied protocol BRUCE on rolling tapestry. The following information was analyzed: age, consumption of tobacco, cardiac frequency basal, PR, axis(axle) QRS, cardiac submaximum frequency, TAS/ TAD basal, maximum TAS/TAD, functional capacity, time of duration of the test(proof), total cholesterol, HDL, LDL and glucose. The cardiovascular risk was analyzed

Results: The average of age was 48,82 years. $29,03 \%$ was smoking. The information analytical was: glycemia 85,37 mg/100 cc, total cholesterol $205 \mathrm{mg} / 100 \mathrm{cc}$, HDL 53,03 mg/100 cc, LDL 151,07 mg/100 cc. Good response to the physical exercise(fiscal year). The functional capacity was 12,87 Mets, and the duration of 11,46 minutes.

Conclusion: To include the ergometry in the protocols of alertness of the health for certain groups of workers

(Med Segur Trab (Internet) 2009; 55 (216): 56-64)

Key words: ergometry, diagnosis, population, firemen 


\section{INTRODUCCIÓN}

La prueba de esfuerzo (PE) o ergometría continúa siendo un procedimiento utilizado en la valoración diagnóstica y pronóstica de los pacientes con cardiopatía isquémica, en estudio o ya diagnosticada. Esta prueba va ampliando su aplicación, y es cada vez más utilizada en otros grupos de sujetos sanos (sedentarios, atléticos, etc) para valorar el estado de resistencia miocárdica al esfuerzo controlado.

El consenso conseguido por el American Collage of Cardiology y el American Heart Association recomienda la prueba siguiendo unas categorías previamente establecidas ${ }^{1}$

- Clase I: existe evidencia y/o acuerdo general en que el procedimiento o tratamiento es útil y efectivo

- Clase II: la evidencia es más discutible y/o existen divergencias en las opiniones sobre la utilidad/eficacia del procedimiento o tratamiento.

- Clase IIa: el peso de la evidencia/opinión está a favor de la utilidad/eficacia.

— Clase IIb: la utilidad/eficacia está menos fundamentada por la evidencia/opinión.

- Clase III: existe evidencia y/o acuerdo general en que el procedimiento o tratamiento no es útil, efectivo y en algunos casos puede ser peligroso.

En cuanto a la metodología de las pruebas de esfuerzo, de las más utilizadas, el cicloergómetro y el tapiz rodante son los de uso más frecuente ${ }^{2}$. Este último es el método más usado, y aunque es más caro, simula un ejercicio más fisiológico y acorde a las necesidades del individuo, no requiriendo ni aprendizaje previo ni entrenamiento. El consumo de oxígeno alcanzado en el tapiz es algo superior al obtenido en la bicicleta ergométrica, aunque la frecuencia cardiaca y la presión arterial son similares en ambos métodos.

La PE es un procedimiento habitualmente seguro (1 fallecimiento por cada 10.000 pruebas realizadas) y lógicamente requiere un consentimiento informado ${ }^{3,4}$.

El protocolo de esfuerzo más utilizado es el de BRUCE, pero lógicamente debemos elegir el más adecuado para cada persona y/o grupo de población de acuerdo con el objetivo que pretendemos con la prueba ${ }^{5-7}$.

Los protocolos que se aplican suelen ser continuos y de intensidad incremental. Frecuentemente el objetivo de la prueba no exige llevar al paciente al esfuerzo máximo. Aunque existe poca correlación entre la edad y la frecuencia cardiaca máxima, la frecuencia cardiaca máxima teórica según edad (220-edad en años) puede ser utilizada como guía.

Dada la dificultad teórica para medir directamente el $\mathrm{VO}_{2}$ en una $\mathrm{PE}$, en la clínica diaria se suele expresar éste en forma de trabajo externo expresado en MET (equivalentes metabólicos) que corresponde a $3,5 \mathrm{ml} / \mathrm{Kg} /$ minuto de $\mathrm{VO}_{2}$ y que permite la comparación entre los diferentes protocolos ${ }^{6,8,9}$.

Los parámetros fundamentales que deben valorarse en la PE se recogen en la TABLA 1.

La realización de ejercicio físico como actividad de ocio o deporte, o incluso la desarrollada como parte de la actividad laboral, someten al organismo a un estrés adicional al habitual de la vida diaria, normalmente sedentaria, y que puede perjudicar o desestabilizar procesos patológicos que contraindiquen dicha práctica deportiva o desarrollo de ciertas actividades laborales.

Las PE deben aportarnos datos que permitan planificar y mejorar nuestro rendimiento físico, tanto en los aspectos deportivos como laborales ${ }^{10-14}$. Por un lado nos servirá para vigilar el estado de salud mediante una prueba que la convierte en un elemento fundamental de prevención secundaria (diagnóstico precoz) y por otro lado nos sirve de apoyo para progresar adecuadamente en el proceso de entrenamiento continuado. 
Tabla 1.

\begin{tabular}{|c|c|}
\hline \multicolumn{2}{|c|}{ Parámetros a evaluar en una PE (prueba de esfuerzo) } \\
\hline Parámetros electrocardiográficos & $\begin{array}{l}\text { Depresión del segmento ST } \\
\text { Elevación del segmento ST } \\
\text { Arritmias y/o trastornos de conducción }\end{array}$ \\
\hline Parámetros hemodinámicas & $\begin{array}{l}\text { Frecuencia cardiaca y presión arterial } \\
\text { Producto de FC x PA sistólica }\end{array}$ \\
\hline Parámetros clínicos & $\begin{array}{l}\text { Angina } \\
\text { Signos de disfunción ventricular izq (mareos, palidez, sudor, } \\
\text { frío, cianosis) } \\
\text { Disnea, claudicación, etc. } \\
\text { Percepción subjetiva del esfuerzo }\end{array}$ \\
\hline Capacidad funcional & $\begin{array}{l}\text { Trabajo externo expresado en MET } \\
\text { Tiempo de ejercicio }\end{array}$ \\
\hline \multicolumn{2}{|c|}{$\begin{array}{l}\text { FC: frecuencia cardiaca. PA: Presión arterial } \\
\text { MET : unidades metabólicas. } 1 \mathrm{MET}: 3,5 \mathrm{ml} \mathrm{de} \mathrm{O}_{2} / \mathrm{Kg} / \text { minuto } \\
\text { Tomado de: Guía Práctica Clínica de la Sociedad Española de Cardiología. } \\
\text { Revista Española de Cardiología. 2000; } 53\end{array}$} \\
\hline
\end{tabular}

Los protocolos a aplicar en población sana, y en la que se intenta valorar su capacidad de ejercicio físico, deben ser incrementales, iniciándose a bajas cargas, con incrementos suaves y progresivos que permitan la adaptación al ergómetro y que sirva de calentamiento. Estas pruebas deben tener una duración óptima de entre 8-12 minutos, y siempre deben ser máximas.

La indicación de PE en deportistas o población trabajadora que desarrolla esfuerzos físicos de moderados a intensos podrían incluirse en la categoría IIa (personas deportistas o con actividad física de moderada a intensa mayores de 35-45 años y con dos o más factores de riesgo cardiovascular. Personas deportistas o con actividad física de moderada a intensa de menos de 35 años con antecedentes familiares de muerte súbita).

Según la American Collage of Cardiology y el American Heart Association (ACC/AHA) 2002, las indicaciones para la realización de ergometrías en sujetos deportistas (o que desarrollen actividades físicas de moderadas a intensas como consecuencia de su actividad laboral) ${ }^{15-16}$ son:

- Valoración de deportistas con sospecha de cardiopatía como indicación de aptitud para la actividad deportiva (clase I)

- Deportistas asintomáticos mayores de 35 años con 2 o más factores de riesgo cardiovasculares (clase II a)

- Orientación sobre el ritmo de competición en deportistas para valorar pruebas de larga duración-resistencia (clase II b)

- Deportistas menores de 35 años para la detección de cardiopatía (clase III).

Con estos antecedentes, desde la Unidad de Salud Laboral del Consorcio de Bomberos de la Provincia de Cádiz, se propone la inclusión en el protocolo de vigilancia de la salud, la realización de una PE a la población trabajadora mayor de 45 años de edad. La indicación se fundamenta en que nos encontramos ante una población laboral que generalmente realiza actividad física deportiva reglada y con un programa personalizado de entrenamiento, y que desarrolla una actividad laboral en la que los esfuerzos físicos puntuales de gran intensidad requeridos, casi siempre, lo son de forma inmediata. 


\section{METODOLOGÍA}

El Consorcio de Bomberos de la Provincia de Cádiz (CBPC) se trata de un Organismo de la Administración Local. Consta de 17 parques distribuidos por toda la provincia, y con una población trabajadora de alrededor de 550 trabajadores/as (mayoritariamente varones)

Los protocolos de vigilancia de la salud que se aplican a la población trabajadora, según la evolución de riesgos realizada por el Servicio de Prevención son: cargas, posturas forzadas, ruido, altura y estrés térmico. A propuesta de la Unidad de Salud Laboral, y con el consenso del Comité de Seguridad y Salud Laboral, se aplica la realización de una PE a la población trabajadora mayor de 44 años, independientemente de la existencia de factores de riesgo cardiovascular. Los datos presentados en este estudio se realizaron durante el año 2007.

La PE se realiza siguiendo protocolo BRUCE, sobre tapiz rodante en sistema MARQUETTE 2000, controlado por sistema HEWLETT PACKARD STRESSWRITTER, con monitorización de doce derivaciones, medidas en tiempo real y toma de constantes vitales cada tres minutos.

Se valoraron las siguientes variables: edad, frecuencia cardiaca basal, PR, eje QRS, frecuencia cardiaca (FC) submáxima, FC máxima, TAS/TAD basal, TAS/TAD máxima, capacidad funcional, tiempo de desarrollo de la prueba, colesterol total, colesterol HDL, colesterol LDL, glucemia basal.

Diseño: Estudio descriptivo transversal

Objetivo: Conocer los resultados de un programa de realización de PE en una población laboral sana mayor de 45 años.

Emplazamiento: Parques de Bomberos de la Provincia de Cádiz.

Participantes: Población trabajadora del Consorcio de Bomberos de la Provincia de Cádiz

\section{RESULTADOS}

La población laboral del Consorcio Provincial de Bomberos de la Provincia de Cádiz es de absoluto predominio masculino (más del 98\% del total). Su media de edad se sitúa alrededor de la treintena (34,58 años con una desviación típica, DE, de 4,91).

En el presente estudio se realizaron un total de 31 Ergometrías (alrededor del 30\% de la población trabajadora de más de 45 años). Todos los sujetos estudiados fueron varones.

Todas las Ergometrías fueron negativas, tanto eléctrica como clínicamente, y tuvieron una buena respuesta de frecuencia cardiaca y de tensión arterial.

La media de edad de la población trabajadora que participó en el estudio fue de 48,82 años (DE 3,57). En cuanto al consumo de tabaco, eran fumadores un total de 9 trabajadores, un $29,03 \%$, con una media de consumo diario de 15,8 cigarrillos/día. Estas cifras son algo superiores al porcentaje de población fumadora en el CBPC, que se sitúa alrededor del $22 \%$.

Los resultados analíticos estudiados nos aportan los siguientes datos de media: Glucemia Basal: 85,37 mg/100 cc (DE 8,21), Colesterol Total: 205 mg/100 cc (DE 18,73), Colesterol LDL: 151,07 mg/100 cc (DE 13,98), Colesterol HDL: 53,03 mg/100 cc (DE 6,91). El análisis del riesgo cardiovascular, en cuanto la perfil lipídico, se expone en la TABLA 2. 
Tabla 2.

\begin{tabular}{lcc}
\hline \multicolumn{2}{c}{ Análisis del Riesgo Cardiovascular. Perfil Lipídico. } \\
\hline Colesterol total & $>250 \mathrm{mg} / 100 \mathrm{cc}$ & 2 \\
& $200-250 \mathrm{mg} / 100 \mathrm{cc}$ & 16 \\
& $<200 \mathrm{mg} / 100 \mathrm{cc}$ & 13 \\
Colesterol HDL & $>35 \mathrm{mg} / 100 \mathrm{cc}$ & 31 \\
& $<35 \mathrm{mg} / 100 \mathrm{cc}$ & 0 \\
Colesterol LDL & $>155 \mathrm{mg} / 100 \mathrm{cc}$ & 11 \\
& $<155 \mathrm{mg} / 100 \mathrm{cc}$ & 20 \\
Riesgo Cardiovascular. Colesterol Total/HDL & Inferior a 3,55 & 3 \\
Riesgo Cardiovascular. Colesterol LDL/HDL & Inferior a 4,50 & 5 \\
\hline Población Laboral del CBPC. Mayores de 44 años de edad. 2007 & & \\
\hline
\end{tabular}

Los datos medios obtenidos de los parámetros valorados en las ergometrías se exponen en la TABLA 3. Cabe destacar la buena respuesta, tanto de la tensión arterial como de la frecuencia cardíaca, al ejercicio realizado. La frecuencia cardíaca máxima alcanzada no superó los 160 latidos/minuto. En cuanto a la tensión arterial, la sistólica no superó los $150 \mathrm{~mm} \mathrm{Hg} \mathrm{y} \mathrm{la} \mathrm{diastólica} \mathrm{los} 90$ mm Hg.

La capacidad funcional demostrada por la población estudiada fue excelente, alcanzando una media de 12,87 METS (DE 1,53). Igualmente la duración media del tiempo empleado en la prueba demostró unos resultados acordes con las características del entrenamiento de la población estudiada, 11,46 minutos de ejercicio (DE 2,18).

En cuanto a las alteraciones del ritmo detectadas en los electrocardiogramas basales, algo más frecuentes en poblaciones entrenadas que en la población general, solamente se detectaron un caso de bloqueo incompleto de rama derecha y un bloqueo aurículoventricular de $1^{\circ}$ grado.

Tabla 3.

Datos de las Ergometrías realizadas (medias y DE)

\begin{tabular}{lc}
\hline Frecuencia cardiaca basal & $71,161 / \mathrm{M}(\mathrm{DE} 3,97)$ \\
PR & $0,173 \mathrm{~mm}(\mathrm{DE} 0,03)$ \\
Eje QRS & $-19,5^{\circ}$ \\
Frecuencia cardiaca submáxima & $148,41 \mathrm{l} / \mathrm{m}(\mathrm{DE} 7,35)$ \\
Frecuencia cardiaca máxima alcanzada & $159,32 \mathrm{l} / \mathrm{m} \mathrm{(DE} \mathrm{6,51)}$ \\
Tensión Arterial Sistólica Basal & $127,30 \mathrm{~mm} \mathrm{Hg}(\mathrm{DE} 10,34)$ \\
Tensión Arterial Diastólica Basal & $78,56 \mathrm{~mm} \mathrm{Hg}(\mathrm{DE} 3,59)$ \\
Tensión Arterial Sistólica Máxima & $148,33 \mathrm{~mm} \mathrm{Hg}(\mathrm{DE} 9,54)$ \\
Tensión Arterial Diastólica Máxima & $89,93 \mathrm{~mm} \mathrm{Hg}(\mathrm{DE} 5,37)$ \\
Capacidad Funcional & $12,87 \mathrm{METS}(\mathrm{DE} 1,53)$ \\
Tiempo de ejercicio & $11,46 \mathrm{minutos}(\mathrm{DE} 2,18)$ \\
\hline Población Laboral del CBPC. Mayores de 44 años de edad. 2007 & \\
\hline
\end{tabular}




\section{DISCUSIÓN}

A pesar de que se conoce la existencia de determinados factores relacionados directamente con el medio laboral, y que de forma independiente o interactuando con otros pueden contribuir al desarrollo, perpetuación o agravamiento de determinadas cardiopatías, pocos sistemas de vigilancia están establecidos para la determinación precoz de cardiopatías en la población trabajadora.

Sin ir más lejos, los Protocolos de Vigilancia de la Salud establecidos por el Ministerio de Sanidad (Comisión Interterritorial del Sistema Nacional de Salud) y que se aplican a la población trabajadora poca referencias hacen a las cardiopatías. Sólo los Protocolos de Posturas Forzadas (abril 2000) y de Movimientos Repetitivos de Miembro Superior (abril 2000) recogen como exploración complementaria a realizar a la población trabajadora expuesta a dichos riesgos la realización de un electrocardiograma basal en reposo. Ni si quiera el Protocolo de Manipulación de Cargas recoge la realización de tal prueba complementaria ${ }^{18}$.

La relación entre la actividad laboral y enfermedades del corazón se había centrado principalmente en la exposición a un grupo reducido de sustancias químicas.

Los factores de exposición en el medio laboral se han ampliado y se clasifican en físicos, químicos, biológicos y psicosociales.

Entre los factores físicos podemos encontrar las modificaciones producidas en la presión parcial de oxígeno, la presión barométrica, la gravedad, la aceleración, la temperatura y la humedad relativa del aire. Los casos de cardiopatía se incrementan en los ambientes fríos ${ }^{19}$. La exposición a vibraciones $(500 \mathrm{hz})$ y a niveles de ruido superiores a los 90 decibelios en el medio laboral se ha demostrado que producen cambios estructurales a nivel pericárdico (aumento de su grosor) ${ }^{20}$. Un metanálisis recoge los resultados de 43 estudios epidemiológicos realizados entre 1970 y 1999 donde se demuestra la relación entre el nivel de ruido (ocupacional o de la comunidad) y la hipertensión arterial y la cardiopatía isquémica ${ }^{21}$. Seguimientos realizados a trabajadores expuestos durante años a ruidos del tráfico demuestran que tienen un mayor riesgo de enfermedades cardiovasculares con un riesgo relativo incrementado en 1,6 (95\% de intervalo de confianza $0,9-3,0)^{22,23}$. Las personas que viven en zonas ruidosas, con niveles superiores a los 65-70 decibelios, incrementan su riesgo de padecer enfermedades cardiovasculares $^{24}$. La población trabajadora que se encuentran expuesta a niveles de ruido que exceden los 90 decibelios tiene un incremento de la frecuencia cardiaca y de la tensión arterial diastólica ${ }^{25,26}$.

La afectación cardiaca por factores de riesgos laborales de origen químico o biológico se centran principalmente en la producción de miocardopatías. Entre los agentes infecciosos podemos encontrar virus (citomegalovirus), bacterias (brucella, clostridium), hongos (aspergilus) o parásitos (toxoplasma, plasmodium). Entre los agentes químicos encontramos el cobalto, plomo, compuestos de antimonio, hidrocarburos halogenados, fósforo, arsénico, mercurio, nitratos alifáticos y disulfuro de carbono, entre otros ${ }^{27}$.

En cuanto a los factores de origen psicosocial son los que se relacionan con el estrés y la turnicidad los que mayor relevancia tienen. El estrés laboral parece ser un predictor independiente de muerte por enfermedades cardiovasculares. Tras el control de los factores de riesgo convencionales, el alto grado de estrés en el trabajo se ha vinculado a una duplicación del riesgo de muerte cardiovascular ${ }^{28}$. Se ha demostrado un riesgo relativo de nuevos infartos de miocardio entre 2 y 4 veces mayor en los trabajadores que experimentan un desequilibrio entre el alto esfuerzo y una baja recompensa. El riesgo relativo de sufrir una enfermedad coronaria, en los 5 años siguientes, es más del doble en los empleados con altos esfuerzos y bajas recompensas que en aquellos sin crisis de gratificación en el trabajo ${ }^{29}$. Los trabajadores que realizan trabajos a turnos tienen en un $40 \%$ incrementado el riesgo de enfermedad cardiovascular. ${ }^{30}$. Existen suficientes evidencias (Evidencia tipo A) que 
relacionan el trabajo a turnos con mayor morbilidad de úlcera gastroduodenal, enfermedades coronarias y problemas en el embarazo ${ }^{31}$.

El diagnóstico precoz de cardiopatía isquémica en personas asintomáticas que desarrollan determinadas actividades laborales puede evitar importantes costes socio sanitarios y reducir las posibles complicaciones mejorando el pronóstico. El objetivo no sólo será prolongar la supervivencia, sino mejorar la calidad de vida ${ }^{32}$.

Son muchos los datos que avalan la inclusión de la ergometría como prueba recomendada en los protocolos de vigilancia de la salud de determinados colectivos laborales.

La Sociedad Española de Cardiología recoge que las personas cuyo trabajo implique seguridad pública, como pilotos de aviones y helicópteros, conductores de camiones y autobuses, conductores de trenes y metros, bomberos, buceadores profesionales, etc., la prueba de esfuerzo permite definir su capacidad funcional y detectar un posible problema coronario ${ }^{17}$.

\section{BIBLIOGRAFÍA}

1. American Collage of Cardiology/American Heart Association Task Force on Assessment of Cardiovascular Procedures: Guidelines for exercise testing. J Am Coll Cardiol 1986; 8:725-738

2. Chaitman B. Las pruebas de esfuerzo. En: Braumwald E, editor. Tratado de Cardiología. Medicina Cardiovascular ( $4^{\mathrm{a}}$ ed). Madrid: McGraw-Hill-Interamericana de España 1993; 177-197.

3. Reyes M, Iñiguez A, Goicolea A, Funes B, Castro A. El consentimiento informado en cardiología. Rev Esp Cardiología 1998; 51:782-796.

4. Fletcher A, Flipse TR, Khigfield P, Malouf JR. Situación actual de la prueba de esfuerzo electrocardiográfica. Curr Probl Cardiol 1999;1:1-125.

5. Howley ET, Bassett JR DR, Welch HG. Criteria of maximal oxygen uptake: review and commentary. Med Sci Sports Exerc 1995; 27: 1291-1301.

6. Wasserman K, Hansen JE, Sue DY, Casaburi R, Whipp BJ. Measurements during integrative cardiopulmonary exercise testing. En: Weinberg R, editor. Principles of exercise testing and interpretation. Baltimore: Lippincott Williams \& Wilkins, 1999; p. 62-94.

7. Fernhall B, Kohrt W. The effect of training specificity on maximal and sub maximal physiological response to treadmill and Cycle Ergometry. J Sport Med Phys Fitness 1990;30:268-75.

8. Borg GA. Psychophysical bases of perceived exertion. Med Sci Sports Exerc. 1982; 14: 377-381

9. Noonan V, Dean E Submaximal Exercise Testing: Clinical Application and Interpretation . Phys Ther. 2000, vol $8, n^{\circ} 8$, august: $782-807$

10. Rich BS. Sudden death screening. Med Clin North Am 1994; 78: 267-288

11. Maroon BJ, Shirin J, Polliac LC, Mathenge R, Roberts WC, Mueler FO. Sudden death in young competitive athletes. JAMA 1996; 276: 199-204

12. Thompson PD. The cardiovascular complications of vigorous physical activity. Arch Intern Med 1996; 156: 2297-2302.

13. Tricoci P, Allen JM, Kramer JM, Califf RM, Smith SC. Scientific evidence underlying the ACC/AHA. Clinical Practice Guidelines. JAMA 2009; 301 (8): 831-841.

14. Dal Monte A. Exercise testing and ergómetros. En: Dirix A, Knuttgen HG, Tittel K, editors. The Olympic book of sports medicine. IOC-FIMS. Oxford: Blackwell Scientific Publications, 1988

15. Gibbons RJ (edit). ACC/AHA 2002 Guidelines Update of Exercise Testing. 2002 American College of Cardiology Foundation and American Heart Association (internet), ACC/AHA; 2002. Disponible en: http:// www.acc.org/clinical/guidelines/exercise/excercise_clean.pdf

16. Guidelines for cardiac exercise testing. ESC Working Group on Exercise Physiology, Physiopathology and Electrocardiography Eur Heart J. 1993; 14: 969-988.

17. Fernando Arós Aros F, Boraita A, Alegría E, Alonso AM, Bardaji A, Lamiel R et al. Guías de práctica clínica de la Sociedad Española de Cardiología en pruebas de esfuerzo. Rev Esp Cardiología 2000; 53 (8): $1063-94$.

18. Protocolos de vigilancia de la salud. Ministerio de Sanidad y Consumo. http://www.msc.es/ciudadanos/ saludAmbLaboral/saludLaboral/vigiTrabajadores/protocolos.htm 
19. Barron HV, Bowlby LJ, Breen T, et al for the National Registry of Myocardial Infarction 2 Investigators. Use of reperfusion therapy for acute myocardial infarction in the United States. Data from the National Registry of Myocardial Infarction 2. Circulation 1998; 97:1150-1156

20. Costelo Branco NA, Alves Pereira M. Vibroacoustic disease. Noise and Health. 2004, vol 6, $\mathrm{n}^{\circ} 23:$ 3-20.

21. Van Kempen EE, Kruize H, Boshuizen HC, Ameling CB, Staatsen BA, de Hollander AE: The association between noise exposure and blood pressure and ischemic heart disease: a meta-analysis. Environ Health Perspect 2002; 110: 307-317.

22. Babisch $W$ et al. Traffic noise and cardiovascular risk: The Caerphilly and Speedwell studies, third phase 10 year follow up. Arch Environ Health. 1999. May-June 54(3):210-216

23. Van Kempen EE, Kruize H, Boshuizen HC, Ameling CB, Staatgen BAM, de Hollander AEM. The association between noise exposure and blood pressure and ischemic hearth disease: a meta-analysis. Environ Health Perspect. 2002 March; 110(3): 307-317

24. Babish W. Traffic noise and cardiovascular disease: Epidemiological Review Noise Health. 2000; 2(8): 9-32

25. Tomei, F; Fantini, S; Tomao, E; Baccolo, TP; Rosati, MV. Hypertension and chronic exposure to noise. Arch Environ Health. 2000 55(5):319-325.Sep-Oct

26. C Reeb-Whitaker, N Seixas, L Sheppard, and R Neitzel. Accuaracy of task recall for epidemiology exposure assessment to construction noise. Occup Environ Med. 2004 February; 61(2): 135-142

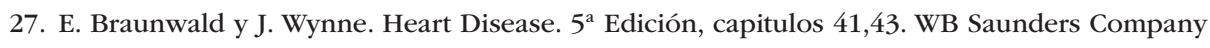

28. Kivimaki M, Leino-Arjas P, Luukkonen R, Riihimaki, H, Vahtera J, Kirjonen J. Work stress and risk cardiovascular mortality: prospectice cohort stady of industrial employees. BMJ 2002: 325, 857

29. Fernández JA, Siegrist J, Rödel A y Hernández-Mejía R. El estrés laboral: un factor de riesgo. ¿Qué sabemos y qué podemos hacer? Atención Primaria 2003. Mayo. Vol 3 N8: 524-526.

30. Boggild H and Knutsson A. Shift work, risk factors and cardiovascular disease. Prim Care. 2000 Dec (4) 1057-79

31. Knutsson A. Health disorders of shift workers. Occupational Medicine. 2003. March 53(2): 103-108

32. Bruce RA, DeRouen TA, Hossack KF. Value of maximal exercise tests in risk assessment of primary coronary heart disease events in healthy men: five years experience of the Seattle heart watch study. Am J Cardiol 1980; 46:371-378.

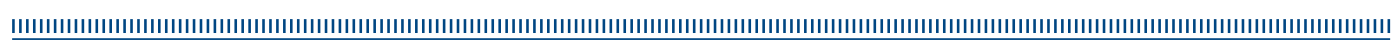

